\title{
Ulrich Hemel
}

\section{Geld oder Leben?}

Risikoethische Fragen rund um die Corona-Krise aus unternehmensethischer Perspektive

\section{Einleitung}

Die Corona-Krise erfordert täglich den verantwortlichen Umgang mit Risiken unterschiedlicher Art: persönlichen, betrieblichen, wirtschaftlichen und anderen. Dass die gesellschaftliche Art, zu leben und zu produzieren, insgesamt gut als >Risikogesellschaft< verstanden werden kann, hat zuerst Ulrich Beck (2003) in seinem vielbeachteten, gleichnamigen Werk beschrieben. In der Zwischenzeit ist die >Risikoethik < zu einem eigenen Gegenstand philosophischer und sozialwissenschaftlicher Betrachtung geworden (vgl. Nida-Rümelin et al. 2012; Hansson 2013; Haltaufderheide 2015).

Dass das Führen von Unternehmen mit Risiken verbunden ist, ergibt sich aus dem Begriff des Unternehmertums. Für diese Risiken steht nicht zuletzt der Gedanke der >schöpferischen Zerstörung (Schumpeter 2005). Häufig genug ist für den einzelnen Unternehmer aber nur die eine Seite, entweder die schöpferische Innovationsfähigkeit oder das Schicksal des Untergangs und Verschwindens vom Markt, als `Zerstörung< erfahrbar.

Die folgenden Überlegungen speisen sich im Sinn des Gesagten aus zwei Quellen: Der unternehmenspraktischen Erfahrung des Autors in der Hochzeit der Corona-Krise und als Inhaber eines Medizinproduktehandels (der Firma Rogg Verbandstoffe) einerseits und der ethischen Reflexion des Erlebten in der Rolle als Direktor des Weltethos-Instituts in Tübingen andererseits (vgl. Hemel 2019).

An entsprechende Beobachtungen schließen sich drei Forderungen an: die nach einer besseren Abbildung von Beschaffungsrisiken im Controlling, die nach einer besseren ethischen Sprachfähigkeit im Management und die nach einer breiteren Diskussion zur Wiedergewinnung des gesellschaftlichen Vertrauens in Unternehmen.

Der plakative Titel dieses Beitrags knüpft an eine Erfahrung aus meiner Kindheit an, das Spiel >Räuber und Gendarm`. Es wurde nur von Jungen gespielt. Wir versteckten uns und überraschten die Mitspieler mit dem Ruf: 'Geld oder Leben`, so wie die damalige Vorstellung von Outlaws und >Räubern< eben war.

Die Klarheit dieser Alternative >Geld oder Leben $<$ bringt die Corona-Krise aus ethischer Perspektive sehr gut auf den Punkt. Es gilt nämlich abzuwägen, welche Risiken Entscheider und Entscheiderinnen einzugehen bereit sind, für sich und für andere. Der elementare Lebensschutz steht in Konkurrenz zu >Geld als dem Sammelbegriff für wirtschaftliches Wohlergehen. 
Unternehmen und Führungskräfte in Unternehmern stehen nicht weniger als andere vor Corona-typischen Entscheidungen, die in ganz anderer Art disruptiv wirken als die Disruption durch digitale Geschäftsmodelle, E-Mobilität und andere. Infrage gestellt wird nämlich ein auf Effizienz ausgerichtetes Modell des Wirtschaftens ohne ausreichendes Risikocontrolling. Von dieser Erkenntnis bis zu ihrer wirtschaftspraktischen Umsetzung ist es aber ein weiter Weg. Dieser Punkt soll weiter unten am Beispiel der Ethik von Lieferketten näher beleuchtet werden.

\section{Drei ethische Herausforderungen}

\subsection{Die Krise beflügelt die Extreme}

In normalen Zeiten herrscht die Verlässlichkeit gegenseitiger Erwartungen, die uns geläufig ist. Es gibt gelernte und eingeübte Prozesse und ein Maß an Veränderung, das im Großen und Ganzen zuträglich ist. In Krisenzeiten gilt dies nicht (vgl. Carrel 2010). Das Tempo der Veränderung ist dann rasant: Was heute gilt, ist womöglich morgen überholt. Dies trifft nicht nur auf politische Entscheidungen zu, sondern auch auf alltagspraktische Themen wie etwa Produktpreise. An Tankstellen sind wir daran gewöhnt, dass Preise täglich schwanken. Bei Medizinprodukten ist ein >Tagespreis für Schutzmasken und Desinfektionsmittel hingegen eine neue Erfahrung.

Ebenso wie im Alltagsleben ganz großartige Formen von Solidarität in der Krise zu beobachten waren, sahen wir auch krassen Egoismus. Dieser zeigte sich in Deutschland beim Horten von Toilettenpapier, in USA beim Ausverkauf von Waffen, in Frankreich durch Hamsterkäufe beim Rotwein. Im zwischenstaatlichen Bereich stellte Deutschland Intensivbetten für Patienten und Patientinnen aus Italien und Frankreich zur Verfügung, war aber auch eines der ersten Länder, das den Export medizinischer Schutzausrüstung blockierte. Dieser nationale Egoismus wurde auch nachgeahmt. So entschied etwa die polnische Regierung, das Desinfektionswerk der Desinfektionsmittel-Produzenten Dr. Schumacher und Norenco Polska mit einem Exportstopp zu belegen. Das führte zu einer weiteren Verknappung und Verteuerung von Desinfektionsflüssigkeit. Noch dreister sind Beispiele aus den USA. RegierungsvertreterInnen tauchten in China an Frachtflugzeugen auf und lenkten die geladenen Medizinprodukte durch Zahlung des dreifachen Preises in die USA um. Auch die in Nordrhein-Westfalen gelagerten Beatmungsgeräte eines bekannten amerikanischen Herstellers wurden blockiert und in die USA exportiert.

Der Wettlauf des Egoismus ist aber nicht auf Staaten begrenzt. Denn selbst wenn dringend benötigte Ware ankommt, ist noch nicht klar, ob die gesetzten Ziele erreicht werden. Auf dem Höhepunkt der Krise ließ sich beispielsweise der bayerische Ministerpräsident Markus Söder auf dem Flughafen München vor einer Frachtmaschine mit acht Millionen Mund-Nase-Bedeckungen fotografieren. 
Das Foto war echt, die Ware leider nicht: Sie musste aus Qualitätsgründen verschrottet werden.

TrittbrettfahrerInnen der Krise gab es auch im Kleinen. Da für den Export von Medizinprodukten in die EU ein sogenanntes CE-Zertifikat benötigt wird, kamen massenweise Fälschungen in Umlauf. Ein CE-Zertifikat wird von autorisierten Qualitätsauditoren im Namen der EU vergeben und ist die Voraussetzung für den Vertrieb von Medizinprodukten. Mehrere Fälschungen konnte der Autor persönlich in Augenschein nehmen, denn ein einziger Qualitätsauditor namens >Rafal K. hat angeblich zwischen Mitte März und Mitte April 2020 Dutzende Fabriken in China zertifiziert, was schon physisch unmöglich ist. Immerhin machte es die immer wiederkehrende gleiche Unterschrift den fachkundigen Einkaufsabteilungen leicht, den Betrug zu erkennen. Doch auch der Verzicht auf ein scheinbar schnelles Geschäft ist ethisch anspruchsvoll: Viele TrittbrettfahrerInnen meinten, auf Qualitätszertifikate verzichten zu können.

Andererseits brachte die Krise einen oft unterschätzten Aspekt zum Vorschein: den Wert langjähriger Kunden- und Lieferantenbeziehungen. Einem langjährigen Lieferanten oder einer langjährigen Lieferantin glaubt man leichter als einem Fremden. Wenn Ware knapp wird und zugeteilt werden muss, sind langjährige treue KundInnen im Vorteil gegenüber noch unbekannten NeukundInnen oder gar TrittbrettfahrerInnen.

\section{2 Überleben ist der Schlüssel}

Es mag auf den ersten Blick erstaunen, wenn das Überleben als ethische Herausforderung gewürdigt wird. Macht man sich allerdings klar, dass Ethik grundsätzlich im Dienst der Förderung von Leben, aber auch von gutem Zusammenleben steht, dann wird das Überleben sehr wohl zu einer ethischen Kategorie. In der Corona-Pandemie gilt dies sogar ganz elementar. Denn alle politischen Einschränkungen wurden ja mit dem Vorrang des Lebensschutzes begründet, und zwar auch dann, wenn einzelne Maßnahmen mit Blick auf das Ziel des >Überlebens geradezu widersprüchlich waren.

So hieß es in Deutschland am Anfang der Krise, das Tragen eines Mundschutzes bringe im Grunde nicht viel, könne sogar durch das Berühren mit infizierten Händen und die Ansammlung von Pathogenen kontraproduktiv wirken. Auch gab es zu Anfang der Krise einen eklatanten Mangel an Mund-Nase-Bedeckungen, sodass der Wunsch verständlich war, die verfügbaren Produkte dem medizinischen Personal zu überlassen. In entsprechender Weise äußerte sich der Bundesgesundheitsminister Jens Spahn.

Ein zweites Beispiel für Widersprüchlichkeit von `Maßnahmen zum Überleben ist die Bandbreite der staatlich verordneten Quarantäne- und Schutzmaßnahmen. Diese reichen von extremen Restriktionen, wie in Spanien und Italien, über eine eher ausgewogene Position, wie in Deutschland, bis hin zur maßvoll gemeinten Herdenimmunität in Schweden. Bisher gibt es in Schweden zwar keinen Lock- 
down der Wirtschaft, wohl aber eine Sterblichkeit, die nach öffentlich verfügbaren Statistiken und dem Stand Mitte Mai 2020 mit 36 Personen pro 100.000 mehr als viermal so hoch ist wie in Deutschland mit 8 pro 100.000 Einwohnern. Wie sich das Bild auf Dauer gestaltet, wissen wir jedoch noch nicht.

Aufgrund persönlicher Kontakte in Ländern wie Peru und Ägypten erhielt der Autor Informationen zu öffentlichen Maßnahmen in jenen Ländern, die sich deutlich vom europäischen Kontext unterschieden. In Peru durften Männer an bestimmten Wochentagen, Frauen an anderen Wochentagen auf die Straße. In Ägypten galt eine Art Halbquarantäne, und zwar abends und nachts von $18 \mathrm{Uhr}$ bis 6 Uhr. Tagsüber galt die Quarantäne nicht. Der Sinn der Regel erschließt sich aus dem ethischen Prinzip des Überlebens: Viele der fast 100 Millionen Menschen in Ägypten sind so arm, dass sie eine strenge Quarantäne mangels Nahrung gar nicht überstehen würden.

Auch für Unternehmen ist das Überleben der Schlüssel. Angesichts massiver Umsatzausfälle in zahlreichen Branchen war hier ein gutes Finanz- und Liquiditätsmanagement entscheidend. Die Ausdehnung des Kurzarbeitergelds und die massiven Finanzhilfen des Staates waren in Deutschland tatsächlich zielführend. Ethisch problematisch ist in diesem Zusammenhang freilich nicht nur möglicher Subventionsbetrug, sondern auch die noch nicht ganz abzuschätzenden Folgen der Finanzhilfen.

Denn einerseits helfen kurzfristige Finanzhilfen schwachen Unternehmen mitten in einer Strukturkrise nur bedingt. Dies kann in der Automobilindustrie und im Maschinenbau ganz unmittelbar beobachtet werden. Andererseits ist die Tatsache, dass gerade starke Unternehmen noch stärker werden, problematisch, da dadurch Markt- und Machtkonzentrationen tendenziell zunehmen.

Was für Unternehmen gilt, gilt auch für Staaten. Wer wie Griechenland und Italien unter einer großen Schuldenlast stöhnt, kann aus eigener Kraft kaum milliardenschwere Aufbauprogramme schultern. Hier kommt die Rolle der EU zum Tragen und die Frage nach Solidarität. Die ethische Frage des Überlebens lässt sich auch hier anwenden: Für Deutschland sind die EU-Partner wichtige Exportmärkte, sodass deren Unterstützung auch im eigenen Interesse liegt. Kommt es hier zu eher kurzfristigen Abwägungen, steigt das Risiko eines Erstarkens populistischer Kräfte, ja des Auseinanderbrechens der Europäischen Union.

Aus globaler Perspektive gelten ähnliche Überlegungen. Staaten mit einer älteren Bevölkerung und einer wenig digital-affinen Wirtschaft werden nach der Krise schlechter dastehen als Staaten mit jüngerer Bevölkerung und digital affiner Wirtschaft.

Die ethische Frage des Überlebens wird an dieser Stelle auch zur Rückfrage an die eingenommene Perspektive. Anders gesagt: Welches Wir gilt? Stehen wir zu unserer Verantwortung als globaler Zivilgesellschaft, die dem Recht auf Leben und Überleben aller Menschen auf der Erde gleichermaßen Wert beimisst? Oder reicht die EU aus, dann aber in einer stärker multipolaren, eher weniger stark globalisierten Welt? 
Wer so denkt, könnte auch zurückgehen auf die Ebene des Nationalstaats, der eigenen Region, der eigenen Kommune, des eigenen Betriebs, der eigenen Familie. Die ethische Frage des Überlebens ist folglich auch eine Frage des Bezugspunkts.

Die Suche nach dem richtigen Bezugspunkt des Handelns ist unter diesem Blickwinkel auch für Unternehmen eine enorme Herausforderung, wie wir am Beispiel der Lieferketten noch zeigen werden.

\subsection{Die Krise ist Stunde der Wahrheit für Führung und Kommunikation}

Was im politischen Raum gilt, gilt auch für Unternehmen: Entscheidend ist gute Führung und gute Kommunikation.

Nun lässt sich trefflich darüber streiten, worin gute Führung besteht. Einige Elemente sind aber durchaus konsensfähig: Es geht um den Abgleich von Prioritäten, um das Herstellen gemeinsamer Sichtweisen und Perspektiven, um die Verständigung über Ziele und Methoden, aber auch um die Herstellung und Erhaltung emotional tragfähiger Beziehungen (vgl. Berner et al. 2015).

Eine besonders markante Beobachtung betraf in der Corona-Krise die spezifisch soziale Komponente von Führung. Menschen möchten angesprochen und wahrgenommen werden. Wo dies besonders gut gelingt, wird die emotionale Bindung der Mitarbeitenden an das Unternehmen gestärkt. Der Autor konnte dies bei einem Betrieb beobachten, indem er im Aufsichtsgremium tätig ist. Es geht um ein produzierendes Unternehmen der Papier- und Schreibwarenindustrie mit knapp 1000 Mitarbeitern und Mitarbeiterinnen. Dieses verschickte Woche für Woche einen >Corona-Newsletter an alle Beschäftigten. Der Inhalt war weitgehend bereits im Vorfeld bekannt. Ergänzt wurde er durch Informationen zu wenig spektakulären internen Maßnahmen. Der Effekt aber war hervorragend: Das Personal fühlte sich informiert, die Führung gewann an Glaubwürdigkeit.

Ein anderes Beispiel betrifft den Umgang mit dem digitalen Raum. Der Autor berichtet an dieser Stelle erneut eine persönliche Erfahrung. In der Corona-Krise wurde an manchen Tagen eine Videokonferenz durch die nächste abgelöst. Doch Telearbeit ist auf Dauer anstrengend, weil die Unmittelbarkeit des sozialen Kontakts fehlt. Paradoxe Abhilfe schaffte hier in einem gegebenen Kontext der wöchentliche >Team-Lunch. Ziel war eine Art des informellen Zusammenseins, eine Art von digitaler Teilhabe, ohne Agenda und vorbestimmte Ziele. Die Teilnahme war freiwillig, aber die meisten Teammitglieder kamen dazu. Denn es handelte sich um eine Maßnahme der wechselseitigen Wahrnehmung, Beachtung und Wertschätzung, bei der auch gefragt werden konnte, wie es der oder dem Einzelnen denn tatsächlich geht.

Daraus ergibt sich auch eine entsprechende Schlussfolgerung: Die denkbar schlechteste Kommunikation in einer Krise ist das Schweigen, wie beispielsweise aus dem Gebiet der Krisenkommunikation bei Sanierungen bekannt ist (vgl. Wilkes/Fleischhauer 2006: 161-166). Wer sich als Führungskraft in der Krise nicht äußert, lässt Raum für Mutmaßungen, Spekulationen oder gar betriebsinterne 
Verschwörungstheorien. Im Zweifelsfall bedeutet es dann mehr Aufwand, die Folgeeffekte spekulativer Kommunikation einzudämmen, statt von Anfang an Klarheit zu schaffen (vgl. Pörksen 2018).

Das Gegenargument, man könne ja nichts mitteilen, weil es nichts Neues gebe, mag sachlich richtig sein, zielt aber ins Leere. Krisenzeiten sind Zeiten erhöhter Unsicherheit. Gute Kommunikation schafft Sicherheit auch dann, wenn sie lediglich wiederholt, etwa nach dem Motto, es gebe diese Woche keine wesentlichen Neuigkeiten, aber die Firma würde zuverlässig informieren, sobald neue Entwicklungen auftreten.

Jede tiefe Krise macht tiefliegende Charakterzüge sichtbar. Ängstliche und unsichere Führungskräfte werden sich in der Krise besonders ängstlich und unsicher zeigen. Wer sich eher am >Laissez-Faire handeln als $>$ Law and Order $<$ Hardliner. In der professionellen Ethik spricht man hier von Laxismus und Rigorismus. ${ }^{1}$ Und wenn Führungskonflikte schwelen, kommen sie in der Krise noch deutlicher zum Ausdruck als zuvor.

Charakterzüge bleiben während der Krise erhalten: Die einen sind stärker von der Angst bestimmt, Fehler zu machen, und verlieren gerade deshalb an Vertrauen. Die anderen leben im Bewusstsein, dass eine gute grundsätzliche Orientierung auch die Korrektur von Fehlern erlaubt. Es kommt durchaus vor, dass sie den eigenen Kurs korrigieren müssen. Dies schadet aber dann nicht, wenn die Gründe dafür erläutert werden, speziell vor dem Hintergrund eines verantwortlichen Faktenchecks.

Die Krise kann dann auch zum Anlass für eine langfristig orientierte Neuausrichtung werden. Die soll in den folgenden Abschnitten unter >Lerneffekte aus der Krise beleuchtet werden. Dabei greife ich als Erstes das Thema des Risikomanagements im Einkauf auf.

\section{Forderungen und mögliche Lerneffekte aus der Krise}

\subsection{Wir brauchen ein besseres Risikomanagement im Einkauf}

Es mag erstaunen, wenn hier ausgerechnet der Einkauf und die Beschaffung von Produkten und Dienstleistungen als mögliches Lernfeld hervorgehoben und ethisch beleuchtet wird. Tatsächlich aber bündeln sich in diesen Funktionen grundlegende Fragen unseres Wirtschaftens. Denn bislang wurde der Einkauf in Unternehmen meist nur als Kostenfaktor gesehen.

1 In der Geschichte der Moraltheologie wurde der Begriff Laxismus für eine Deutung von Gesetzen verwendet, die deren Verpflichtungscharakter durch Kritik an ihrer Legitimation abschwächen (»lex dubia non obligat «); der Begriff wird aber meist polemisch zur Herabsetzung der Gegner gebraucht. Der Gegenbegriff ist der Rigorismus, der eine sehr strenge Auslegung befürwortet, manchmal aber auch mit Immanuel Kants Pflichtenethik in Verbindung gebracht wird, weil nur pflichtgemäßes Handeln legitim und sittlich gut sei (vgl. Demmer 1989; Kleber 1994; ferner W Windisch 1992 ). 
Das ist zwar richtig, verweist aber auch auf ein ethisches und ökonomisches Dilemma. Ich möchte noch einmal auf das oben angeführte Beispiel der Schutzmasken zurückkommen. Bis zum Beginn der Corona-Pandemie war das Wort >Alltagsmaske< quasi unbekannt. Aufgrund der geltenden EU-Gesetze wurden derartige Produkte generell als Medizinprodukte angesehen, unterlagen also auch der entsprechenden Regulierung. Die zeitweilige Versorgungsknappheit führte dann zu einer Wiederentdeckung textiler Schutzmasken, die zuvor längst außer Gebrauch geraten waren. Die politische Beurteilung persönlicher Schutzausrüstung, die nicht als Medizinprodukt ausgelegt ist, änderte sich schnell; und plötzlich entstanden textile Alltagsmasken in modischem Design, als persönliches Accessoire, ohne nähere Betrachtung des möglichen medizinischen Nutzens.

Gleichzeitig wollten zahlreiche chinesische Hersteller medizinische Schutzmasken nur noch nach Vorkasse liefern. Zahlung per Vorkasse aber setzt erhöhtes Vertrauen voraus und ist mit Risiken verbunden. Wer es konnte, bevorzugte folglich regionale Lieferanten. Die Schutzwirkung der >Rechtssicherheit`im demokratischen Rechtsstaat war dabei ein unterstützendes Argument.

Für eine klassische Einkaufsabteilung stand die Welt nun Kopf. Die klassischen Parameter - wie Menge, Preis, Wiederbeschaffungszeit und in Abhängigkeit davon der optimale Warenbestand - traten scheinbar außer Kraft. Tatsächlich gab es beim späteren Sinken des >Tagespreises` durchaus einige Marktteilnehmer, die sich zu teuren Preisen bevorratet hatten und diese Vorräte allenfalls unter Verlust losschlagen konnten. Vermehrt trat aber auch die Frage auf, ob denn die Versorgungssicherheit nicht eine größere Rolle als bisher spielen sollte. Krankenhäuser, die eigentlich eine Pflicht zur Vorsorge für den Notfall kennen, verwiesen auf fehlenden Lagerplatz und mangelnde Mittel. Erfahrene EinkäuferInnen in Firmen blieben nach den Erfahrungen des Autors zurückhaltend, denn nach ihrer Meinung sind solche Erkenntnisse schnell vergessen, wenn der Markt wieder snormak funktioniert: Dann zählen erneut ausschließlich Kosten.

Das >Risikomanagement ‘ des Einkaufs ist nämlich in den heutigen Instrumenten des Controllings und der Kostenrechnung nur selten gut abgebildet (vgl. Lattwein/ Roth 2007: 209-230). Das implizite Dogma der ökonomischen Effizienz vernachlässigt systematisch externe Effekte wie den $\mathrm{CO}_{2}$-Fußabdruck, den Wasserrucksack $^{2}$ und andere Fragen aus einer Ethik der Lieferkette. Unterschätzt und unterschlagen werden aber auch die Risiken einer Lieferketten-Unterbrechung, so wie wir das am Beispiel medizinischer Schutzausrüstung in der Corona-Pandemie erlebten. In der Folge der Krise geht es nicht nur um geschlossene Grenzen, sondern auch um den insolvenzbedingten Ausfall von Lieferanten. Das Risikomanagement des Einkaufs muss also idealerweise auch das Ausfallrisiko eines Liefe-

2 Das Konzept des Wasserrucksacks, auch virtuelles Wasser oder Wasserfußabdruck genannt, wurde entwickelt, um eine Aussage darüber zu treffen, wie viel Wasser zur Herstellung von Gütern enforderlichist (vgl. Mausen 2007; Relleret. al. 2013: 53). 
ranten bewerten so wie bei den vom Strukturwandel geplagten Automobilzulieferern und Maschinenbauern.

Bereits heute gibt es hier das Konzept des Dual Sourcing, also des Aufbaus alternativer Lieferanten. Schwierig wird es dort, wo einer von beiden nicht zu den gleichen Preisen wie der andere liefern kann. Soll der Einkauf dann Kostennachteile in Kauf nehmen?

Bisher war die Antwort in den allermeisten Fällen ein klares Nein. Einkaufsabteilungen in Krankenhäusern werden bis heute generell an ihren Kosten gemessen. Als >Cost Center ist der Einkauf grundsätzlich gehalten, zum günstigsten Preis zu beschaffen. Es gab und gibt zwar eine Lieferantenbeurteilung, aber der Preis (oder genauer: die Gesamtkosten der Beschaffungseinheit, inklusive Transport und Regiekosten des Einkaufs, die Total Landed Cost genannt werden) war häufig doch das ausschlaggebende Kriterium.

Die ökonomische Quantifizierung von Beschaffungsrisiken steht in der Praxis noch am Anfang. Im Finanzbereich ist es längst üblich, die Kosten der Währungssicherung bei einem Kurssicherungsgeschäft konsequent zu erfassen. Wenn ein Unternehmen Zahlungen in den Dollarraum leistet, muss es wissen, mit welchem durchschnittlichen Wechselkurs aufs Jahr verteilt zu rechnen ist. Denn andernfalls hinkt die gesamte Preiskalkulation des Unternehmens.

Analog dazu gilt es nun, 'Kosten der Beschaffungssicherheit‘ als eigene Kostenart ins Controlling einzuführen. Dies gilt selbst dann, wenn eine absolute Sicherheit nie gegeben ist, dafür aber Annahmen über Risiken ausdrücklich offengelegt werden. Es handelt sich dann im engeren Sinn um strategische Kosten, die im Finanzbereich eigens erfasst werden müssen. Wer also aus guten Gründen 120 Euro statt 100 Euro für die Beschaffung eines Produkts aufwendet, der würde künftig den Vergleichswert >100 Euro< als Basispreis, die verbleibenden >20 Euro< als >Risikokosten< guter Beschaffung ausweisen.

Gute Beschaffung umfasst dann alle Funktionen eines strategischen Einkaufs: Das Preis-Leistungs-Verhältnis des Produkts, Nebenkosten aus Transport und Beschaffung, aber auch Kosten der Liefersicherheit, der ökologischen Überlegenheit und Aufwendungen aus Fragen rund um die Ethik der Lieferketten inklusive einer Bewertung der benötigten Rohstoffe (vgl. Reller et al. 2013).

Die Quantifizierung dieser Effekte ist anspruchsvoll und nur mit strategischen Annahmen darstellbar. Aber erst der eigenständige Ausweis der Position `Kosten der Beschaffungssicherheit< erlaubt ein selbstständiges Risikomanagement im Einkauf, das auf die heutigen Fragen von Ausfallrisiken, ökologischer Qualität und ethischen Fragen der Beschaffung handlungsrelevant eingehen kann.

Damit einher geht eine Kritik der bisherigen Theorie und Praxis der Wirtschaftswissenschaften, die sich dafür in bis dato ungewohnter Weise normativen und ethischen Fragestellungen öffnen müsste (vgl. Günther/Ruter 2011; Rogall 2015). Das bedeutete nicht das Ende wirtschaftswissenschaftlicher Rationalität oder gar der gesamten Globalisierung in der Lieferkette, sondern eher deren Berei- 
cherung durch die Einbeziehung qualitativer Maßstäbe als Folge einer globalen Pandemie, einem echten >Moment of Truth .

\subsection{Wir brauchen eine bessere ethische Sprachfähigkeit im Management}

Veränderungen bedürfen intensiver Kommunikation. Für die meisten Unternehmenslenker und Manager oder Managerinnen geht es hier um eine in diesem Ausmaß neue Herausforderung. Noch vor 20 Jahren genügte es als CEO, gute Zahlen und eine nachvollziehbare Strategie vorzulegen. Heute erfordert es der gesellschaftliche Konsens, auch die eigene Werteorientierung auszuweisen und verständlich zu kommunizieren. Ethische Unternehmenskommunikation ist aber kein Selbstläufer, sondern ein komplexer Lernprozess. Die kommunikativen Herausforderungen, die sich aus dem Bau einer Signalanlage für ein Kohlekraftwerk in Australien für den Siemens-Chef Joe Kaeser ergaben, können das Gesagte illustrieren. In der Corona-Krise gab es ein ähnliches Beispiel bei der Kommunikation des Adidas-Chefs Kasper Rorsted zur Aussetzung von Mietzahlungen just, nachdem für das vergangene Jahr 2019 ein Rekordgewinn in Höhe von 2,8 Milliarden Euro erzielt worden war.

Ethische Sprachfähigkeit fällt nicht vom Himmel. Sie muss gelernt und eingeübt werden. Sie benötigt insbesondere den Brückenschlag zwischen Theorie und Praxis. Besondere Aufmerksamkeit verdienen daher entsprechende Versuche, etwa die Förderung von >Weltethos-Ambassadors < durch das vom Autor geleitete Weltethos-Institut in Tübingen (vgl. Hemel 2019). In diesem Zusammenhang wurde unter anderem ein wahrnehmungsbasiertes >Ethisches Rating von Unternehmen entwickelt, das eine Hilfestellung zur Entwicklung ethischer Sprach- und Handlungsfähigkeit von Unternehmen darstellt.

Ethische Sprachfähigkeit hat insbesondere den Zweck, die Fähigkeit zur Übernahme von Verantwortung mit der Anschlussfähigkeit zum zivilgesellschaftlichen Diskurs zu verbinden. Wie in vielen anderen Fällen ist die Corona-Krise hier ein Katalysator zur Beschleunigung von Entwicklungen, die sich auch zuvor schon Bahn gebrochen hatten. Schon mit Blick auf ihr Talentmanagement müssen Unternehmen heute glaubhaft darlegen können, wie sie Wertschöpfung mit Sinnschöpfung verbinden. Mehr und mehr wird von ihnen gefordert, genauer als bisher darzulegen, welchen gesellschaftlichen Beitrag sie jenseits guter Gewinnzahlen erbringen können. Was passiert, wenn es an der Verknüpfung von Verantwortung und ethischer Sprachfähigkeit fehlt, lässt sich am Beispiel der >Deutsche Wohnen AG in Berlin betrachten. Dort kam es, nicht zuletzt als Folge eher ungeschickter Unternehmenskommunikation, zu öffentlichen Demonstrationen, die sich $-\mathrm{zu}$ Recht oder zu Unrecht - gegen ein einzelnes Unternehmen richteten und im Ruf nach >Vergesellschaftung gipfelten. Anders ausgedrückt: Die eklatante Unfähigkeit $\mathrm{zu}$ ethischer Unternehmenskommunikation ist ein so hohes unternehmerisches Risiko, dass es in der Risikolandkarte jedes Unternehmens abgebildet werden sollte. 
Wie der Hinweis auf die Praxis im Weltethos-Institut zeigt, sollte die Förderung ethischer Sprachfähigkeit im Management kein isolierter Appell bleiben, sondern in einen gemeinsamen Lernprozess münden, der sowohl die Wirtschaftswissenschaften als auch die Unternehmenspraktiker umfasst. Die Förderung ethischer Sprachfähigkeit ist Teil der Risikominimierung bezüglich der gesellschaftlichen Akzeptanz eines Unternehmens. Denn die ethischen Fragen rund um den Zugang zum Gesundheitswesen, die ökologische Qualität von Wertschöpfung und den gesellschaftlichen Beitrag von Unternehmen werden in den nächsten Jahren weiter an Bedeutung gewinnen.

\subsection{Gesellschaftliches Vertrauen und eine neue Art des Wirtschaftens sind der Schlüssel zur Zukunft}

Die Corona-Krise hat im wirtschaftlichen und gesellschaftlichen Bereich erst begonnen. Manches spricht dafür, dass sie tiefer ist und länger dauert, als wir das zum Zeitpunkt des Abfassens dieses Beitrags, im Frühsommer 2020, erwarten. Wer den Schub für die digitale Transformation, für Videokonferenzen und Homeoffice erlebt hat, der wird weniger Dienstreisen planen als früher. Die Auswirkungen daraus betreffen beispielsweise den Flugverkehr: Möglicherweise bezeichnet das Jahr 2019 den >Peak of Air Traffic mit erheblichen Folgen für Fluglinien wie die Lufthansa und andere, die staatliche Hilfe zur Rettung vor der Insolvenz benötigten. Aber auch die Automobilindustrie wird einen noch stärkeren Druck in Richtung Strukturwandel erleben, mit erwartbaren Effekten für Geschäftsmodelle und Arbeitsplätze.

Die massive Kurzarbeit und der Anstieg der Arbeitslosigkeit wirken sich auf das Verbrauchervertrauen aus, sodass eine schnelle Erholung der Konjunktur auch aus diesem Grund weniger wahrscheinlich erscheint als zu Beginn der Krise.

Die zum Teil noch massiveren Einbrüche in anderen europäischen Ländern werden zugleich die Wirtschaftsaussichten einer auf Export ausgelegten Wirtschaft wie in Deutschland trüben. Sinkende Gewinne und Steuereinnahmen werden die staatliche Schuldenkrise, aber auch den Zusammenhalt in Europa vor neue Herausforderungen stellen. Trotz populistischer Tendenzen liegt die langfristige Antwort nur in einer Vertiefung der europäischen Zusammenarbeit, etwa durch eine an die nationale Kaufkraft gekoppelte Arbeitslosenversicherung sowie einen gemeinsamen Korridor der Unternehmensbesteuerung. Im Bereich der Umsatzsteuer, die auf eine Bandbreite zwischen 16 und 25 Prozent in Europa festgelegt ist, sind solche Spielregeln schon bekannt.

Digitale, ökologische, regionale und ethisch sprachfähige Geschäftsmodelle werden zu den Gewinnern der Corona-Pandemie gehören. Ein reines >Weiter so ist unwahrscheinlich. Die Corona-Krise ist mit Blick auf die verengte Auslegung des Wirtschaftens auf den Wert der funktionalen Effizienz eben auch ein Spiegelbild der bisherigen Art und Weise des Wirtschaftens, die wesentliche Bereiche eines umfassenden Risikomanagements außer Acht gelassen hatte. 


\section{Schluss}

Wenn es tatsächlich Lerneffekte aus der Krise geben soll, muss ein solches umfassendes Risikomanagement vom Einkauf bis zur ethischen Unternehmenskommunikation erst noch umgesetzt werden. Zu dieser Umsetzung gehören auch Strukturen im Controlling und in der ethischen Diskursfähigkeit von Führungskräften (vgl. Hemel 2007: 53-62). Denn erst die Kombination aus Wertschöpfung und Sinnschöpfung, aus Eigennutz und gesellschaftlichem Wohlergehen, aus ökonomischem Zweck und übergreifendem Sinn schafft eine Zukunft, die auch für die kommenden Generationen lebenswert sein wird.

\section{Literaturverzeichnis}

Beck, U. (2003): Risikogesellschaft. Auf dem Weg in eine andere Moderne, Frankfurt/Main: Suhrkamp.

Berner, W./Hagenhoff, R./Vetter, Th. (2015): Ermutigende Führung, Stuttgart: Schäfer-Poeschel.

Carrel, L. (2010): Leaderhip in Krisen. Ein Leitfaden für die Praxis, Wiesbaden: Gabler Springer.

Demmer, K. (1989): Moraltheologische Methodenlehre, Freiburg: Herder/Universitätsverlag Freiburg.

Günther, E./Ruter, R. X. (2012): Grundsätze nachhaltiger Unternehmensführung, Berlin: E. Schmidt.

Haltaufderheide, J. (2015): Zur Risikoethik, Analysen im Problemfeld zwischen Normativität und unsicherer Zukunft, Würzburg: Königshausen \& Neumann

Hansson, S. O. (2013): The Ethics of Risk. Ethical Analysis in an Uncertain World, New York, London: Palgrave Macmillan.

Hemel, U. (2007): Ethikcontrolling. Wertschöpfung durch Wertesteuerung, in: Horváth, P. (Hrsg.): Erfolgstreiber für das Controlling, Stuttgart: Schaefer Pöschel, 53-62.

Hemel, U. (2019): Weltethos für das 21. Jahrhundert, Freiburg/Breisgau: Herder.

Horváth, P. (2007): Erfolgstreiber für das Controlling, Stuttgart: Schäfer-Poeschel.

Kleber, K. H. (1994): Einführung in die Geschichte der Moraltheologie, Passau: Passavia-Universitätsverlag.

Lattwein, J./Roth, M. (2007): Finanzwirtschaftliches Risikocontrolling der Supply Chain, in: Horváth, P. (Hrsg.): Erfolgstreiber für das Controlling, Stuttgart: Schaefer Pöschel, 209_ 230.

Mauser, W. (2007): Wie lange reicht die Ressource Wasser? Vom Umgang mit dem blauen Gold, Frankfurt/Main: Fischer.

Pörksen, B. (2018): Die große Gereiztheit. Wege aus der kollektiven Erregung, München: Hanser.

Reller, A./Marschall, L./Meissner, S./Schmidt, C. (2013): Ressourcenstrategien, Eine Einführung in den nachhaltigen Umgang mit Ressourcen, Darmstadt: wbg.

Rogall, H. (2015): Grundlagen einer nachhaltigen Wirtschaftslehre, Marburg: Metropolis.

Nida-Rümelin, J./Rath, B./Schulenburg, J. (2012): Risikoethik, Berlin, Boston: De Gruyter.

Schumpeter, J. A. (2005): Kapitalismus, Sozialismus und Demokratie, Stuttgart: UTB.

Smith, R. (2002): Global Supply Chain Performance and Risk Optimization, Wiesbaden: Gabler Springer. 
Wilkes, M. W./Fleischhaner, A. (2006): Kommunizieren wenn es drauf ankommt! In der Not frisst der Teufel Fliegen. Der Manager löst das Problem professionell, Berlin: Erich Schmidt Verlag.

Windisch, H. (1992): Rigorismus, in: Schütz, C. (Hrsg.): Praktisches Lexikon der Spiritualität, Freiburg/Breisgau: Herder, Spalte 1061-1062. 